\title{
The cost of procuring deceased donor kidneys: Evidence from OPO cost reports 2013-2017
}

\author{
Philip J. Held ${ }^{1}$ (D) | Jennifer L. Bragg-Gresham ${ }^{2}$ | Thomas Peters ${ }^{3}$ | Glen M. Chertow ${ }^{4}$ | \\ Frank McCormick $^{5}$ (D) | John P. Roberts ${ }^{6}$
}

${ }^{1}$ Department of Medicine - Med/ Nephrology, Stanford University, Stanford, California

${ }^{2}$ Department of Internal Medicine Nephrology, University of Michigan, Ann Arbor, Michigan

${ }^{3}$ Department of Surgery, University of Florida, Jacksonville, Florida

${ }^{4}$ Department of Medicine - Med/ Nephrology, Health Research \& Policy, Stanford University, Stanford, California

${ }^{5}$ Independent Consultant, Walnut Creek, California

${ }^{6}$ Department of Surgery, Division of Transplant Surgery, University of California San Francisco, San Francisco, California

Correspondence

Philip J. Held

Email: esrd00@gmail.com
Using 5 years of US organ procurement organization (OPO) data, we determined the cost of recovering a viable (ie, transplanted) kidney for each of 51 OPOs. We also examined the effects on OPO costs of the recovery of nonviable (ie, discarded) kidneys and other OPO metrics. Annual cost reports from 51 independent OPOs were used to determine the cost per recovered kidney for each OPO. A quadratic regression model was employed to estimate the relationship between the cost of kidneys and the number of viable kidneys recovered, as well as other OPO performance indicators. The cost of transplanted kidneys at individual OPOs ranged widely from $\$ 24000$ to $\$ 56000$, and the average was $\$ 36000$. The cost of a viable kidney tended to decline with the number of kidneys procured up to 549 kidneys per year and then increase. Of the total 81401 kidneys recovered, 66454 were viable and 14947 (18.4\%) were nonviable. The costs of kidneys varied widely over the OPOs studied, and costs were a function of the recovered number of viable and nonviable organs, local cost levels, donation after cardiac death, year, and Standardized Donor Rate Ratio. Cost increases were 3\% per year.

\section{KEYWORDS}

donors and donation: deceased, economics, ethics and public policy, health services and outcomes research, kidney transplantation/nephrology, organ procurement and allocation, Organ Procurement and Transplantation Network (OPTN), organ procurement organization, organ transplantation in general

\section{1 | INTRODUCTION}

The 58 US organ procurement organizations (OPOs) are not-forprofit entities with a federal contract to cover a specific geographic area over which the OPO is granted exclusive responsibilities, that is, a monopoly. Required OPO tasks include assessment of potential organ donors, obtaining consent for organ donation from next of kin, surgical recovery and preservation of viable organs, and transport of organs to transplant center hospitals. Allocation of any organ

Abbreviations: CMS, Centers for Medicare and Medicaid Services, a part of the US Department of Health and Human Services; COL, cost of living; DCD, donation after cardiac death; NOTA, National Organ Transplantation Act; OAC, organ acquisition costs; OPO, organ procurement organization; OPTN, Organ Procurement and Transplantation Network. to a specific recipient is directed through the Organ Procurement Transplantation Network (OPTN) and the United Network for Organ Sharing (UNOS), which holds the federal contract from the OPTN. Transplant center professionals make the final decision to accept and transplant or reject and discard any individual organ.

Whereas the costs for most aspects of the organ transplant process have been reported, ${ }^{1-3}$ the functional OPO cost, generally referred to as the organ acquisition cost (OAC), has not been widely researched. ${ }^{4}$ As a consequence of the National Organ Transplantation Act (NOTA), ${ }^{5}$ OPOs have a defined method of assessing OAC, and the aggregate costs incurred by any OPO are unique to that OPO. Because all areas of the United States are the responsibility of some OPOs, there are many potential variations in 
expenses including local labor costs, the number of potential and actual donors, the number of transplant hospitals in the OPO service area, hospital charges for maintaining donor organ function after brain death (prior to organ recovery), and other costs.

Among US OPOs, the number of kidneys recovered annually varies from fewer than 100 to over 800 kidneys procured and transplanted. In this analysis, we have sought to describe variations in kidney procurement costs across the United States and differences in kidney cost by OPO size (hereafter defined by the number of kidneys procured annually). Kidney acquisition costs related to transplanted (viable) organs and of those related to discarded, "nonviable" kidneys (ie, organs recovered for transplantation but not transplanted) on cost and OPO outcomes were also examined. Understanding cost variations among US OPOs could allow for pragmatic assessments regarding OPO efficiency, economies of scale, and other matters related to policy issues affecting OPOs and the patients ultimately served.

\section{2 | METHODS AND DATA}

\section{1 | Data}

Of the 58 US OPOs, six are hospital based, and specific OPO cost data were not available. Annual cost reports from the remaining 51 independent OPOs were analyzed with data from Form CMS 216$94^{6,7}$ for the 5 years 2013 through 2017. The maximum possible OPO annual reports would be 255 (5 years $\times 51$ OPOs); however, only 40 reports for 2013 and 48 for 2017 were available resulting in a working sample of 241 (95\%) OPO annual reports for the study period.

Unless noted otherwise, the number of counted kidneys is for "viable" organs, which are those kidneys that are ultimately transplanted (Worksheet S-1; Part 1, Line 3; Column 3; Form CMS 21694; all viable kidneys, local and imported). OPO costs incurred in the procurement and sale of tissues are removed by the Centers for Medicare and Medicaid Services (CMS) from their general accounting system, which focuses exclusively on organs via Worksheet B-1 and are excluded from this analysis. Organ procurement direct costs (eg, hospital charges, surgeon fees etc) are aggregated by organ type (kidney, liver, etc) and indirect costs (overhead) are aggregated across all organ types and allocated as a linear function of the sum of organ counts of all types. When multiple organs are removed from a donor, those costs specific to an organ are assigned to the direct cost for that organ, whereas other direct costs are divided equally by the number of organs from that donor.

Costs were calculated as the total expenses an OPO incurred for kidney procurement through delivery to a transplant center. These costs included all direct costs as well as allocated indirect costs. Total costs for kidney procurement within an OPO were divided by the number of viable kidneys to obtain cost per kidney for that OPO and then in the case of the simple mean model averaged for the years of available data with a resulting cost per kidney procured for that OPO. In the repeated measures model (which is the primary model in this manuscript) the cost data were analyzed on a yearly basis.

We adjusted input labor costs by the local price index for each OPO headquarters city using the Expatistan ${ }^{8}$ index, which is a cost of living (COL) database comparing cities worldwide. A second adjustment of local input costs used the CMS Wage Index ${ }^{9}$ for the year 2015 for the county in which the OPO is headquartered.

We examined OPO performance measures including the Standardized Donation Rate Ratio (SDRR) as reported for each OPO by the Scientific Registry of Transplant Recipients. ${ }^{10}$ Because there is controversy regarding this measure, we also examined donors per 1000 deaths of patients under 75 years of age who were likely possible donors (data limited to calendar year 2015) as a potential OPO performance measure recognizing the importance of standardized, verifiable, and objective metrics of OPO performance. ${ }^{11}$

\subsection{Statistical analysis}

For all OPO years, descriptive statistics for cost of kidneys and potential predictors of cost included the following: number of transplanted kidneys, two COL measures, percentage of nonviable kidneys, percentage of donation after cardiac death (DCD) donors, 25th percentile of wait times (in months), number of transplant programs in the OPO, percentage of donors $>65$ years of age, SDRR, and donors per 1000 deaths under age 75 (year 2015). Two statistical models employing linear regression were examined. In model 1 , we averaged the total cost of kidney acquisitions and number of viable kidneys recovered over the 5 years resulting in a single measure of cost and a single measure of viable kidney count for each OPO. In model 2 we used a repeated measures approach in which we considered each set of annual OPO data as a separate observation and used generalized estimating equations to account for the correlation between repeated OPOs, employing a compound symmetry covariance matrix. Results proved robust between the two models, so with a few exceptions as noted, only the repeated measures estimates (model 2) are reported.

These models examined the relations among selected covariates, where cost per kidney was the dependent variable. The independent variable of average number of kidneys procured was examined in linear, squared, and cubic form, after Dor et al. ${ }^{12}$ Other covariates included percentage of nonviable kidneys, year, percentage of donors $>65$ years of age, measures of wait times, number of transplant programs in the OPO, and donors per 1000 deaths age less than $75 .{ }^{11}$ Models adjusted for local input costs using either the COL index ${ }^{8}$ or the CMS Wage Index. ${ }^{9}$ Because results were virtually identical with either adjustment, estimates are displayed only for the COL index adjusted model.

The cost curve of expected cost per kidney by count of kidney transplants is displayed unadjusted, adjusted to the mean $\mathrm{COL}$ index (157.6), and to three levels of percentage of nonviable kidneys (the 5 th percentile, median, and 95th percentile) to display the potential 
role these variable play in determining cost of a kidney. In effect, this estimation adjusted each OPO's cost to the mean (average) of input cost levels to allow for comparison as if all OPOs faced the same input cost level.

Due to the missing values in 2013 and 2017, we performed a sensitivity analysis examining only those OPOs with complete data for all 5 years $(n=40)$ to ensure that results were consistent. No meaningful differences were found in this subanalysis.

\section{RESULTS}

There is wide variation in costs per organ and the number of viable kidneys procured among US OPOs (Table 1). The average cost of a transplanted kidney over the 5-year period was \$36 027 (range: \$24 231-\$55 995; interquartile range [IQR] was from \$31 607 to $\$ 40$ 319) and the average number of kidneys transplanted per OPO was 274 (range: 48-931; IQR = 127-361) across the 238 OPO-years. The number of nonviable kidneys averaged $17.8 \%$. Fifty percent of OPOs (using IQR) recovered between 37 (13.5\%) and 60 (21.9\%) nonviable kidneys per year. The average OPO retrieved 44 DCD kidneys per year and $50 \%$ of OPOs (using IQR) would have retrieved between 27 and 58 DCD kidneys annually.

The number of nonviable kidneys procured was positively associated (slope $=0.2438$ with the number of viable kidneys procured (Figure 1), with an $R^{2}=.74, P<.0001$ ). By simple linear regression, the model suggests that for every 10 viable kidneys there is an expectation that 2.4 nonviable kidneys are recovered.

Figure 2 depicts the OPOs with the most and least costly kidneys. A total of 13 OPOs reported procurement costs below $\$ 32000$ and 12 OPOs reported procurement costs above $\$ 40000$ per transplanted kidney. (These statistics are from the simple mean for each OPO for the 5 years.) The nine donor service areas with the costliest kidneys included Buffalo, NY; North Liberty, IA; San Ramon, CA; Honolulu, HI; Los Angeles, CA; Las Vegas, NV; Troy, NY; Greenville, NC; and Bloomfield, CT. The seven OPO service areas with the least costly kidneys included Tampa, FL; Indianapolis, IN; North Charleston, SC; Nashville, TN; Pittsburgh, PA; San Antonio, TX; and Philadelphia, PA.

Table 2 presents the basic estimated least squares models with the same dependent variable of average cost per viable kidney. Mean cost per kidney across OPOs $=\$ 36102, \mathrm{n}=234$ The $R^{2}$ is .32 with 237 observations in this repeated measure model clearly suggesting a quadratic relationship with the coefficient on the count of organs procured and organs squared being substantial and statistically significant. Addition of a cubic term to a model with linear and quadratic terms failed to improve the model fit. The full model contains 14 covariates that include a range of measures reporting on a series of hypotheses regarding OPO operations. The left side of Table 2 presents the results for this full model and the right side presents the model limited to the 7 of 14 covariates that passed the test of significance at $P<.05^{13}$

The results indicate that OPOs in areas of the country with higher COL have higher kidney costs $(+\$ 94.89$ higher cost per kidney per 1-unit higher $\mathrm{COL}, P=.002$ ). OPOs located at the 75 th percentile of COL would have an average higher cost per kidney of \$2087 than OPOs at the 25th percentile of COL.

OPOs that retrieved more nonviable kidneys experienced higher cost per viable kidney. As shown in Table 2 (right side), in comparing OPOs with the same number of procured kidneys, having $1 \%$ more nonviable kidneys is associated with a $\$ 275$ higher cost per viable kidneys. Comparing the IQR for percentage of nonviable kidneys, which is $7.7 \%$ (21.2\%-13.5\%), translates into an increased cost of $\$ 2121$ per viable kidney (ie, comparing an OPO at the 75th percentile of percentage of nonviable vs the 25 th percentile of percentage of nonviable kidneys).

TAB LE 1 Basic sample/census statistics, organ procurement organization (OPO) unit of observation, 2013-2017

\begin{tabular}{|c|c|c|c|c|c|}
\hline Variable & Mean & SD & Interquartile range & Minimum & Maximum \\
\hline Average cost per kidney/OPO & $\$ 36026$ & $\$ 5989$ & $\$ 31607-\$ 40319$ & $\$ 24231$ & $\$ 55995$ \\
\hline $\begin{array}{l}\text { Cost of living in the city where OPO is } \\
\text { headquartered }\end{array}$ & 157 & 24 & $142-164$ & 124 & 239 \\
\hline $\begin{array}{l}\text { Centers for Medicare and Medicaid } \\
\text { Services Wage Index in the county } \\
\text { where OPO is headquartered }\end{array}$ & 1.01 & 0.17 & $0.90-1.04$ & 0.82 & 1.71 \\
\hline Nonviable organs (\%) & 17.8 & 5.7 & $13.5-21.2$ & 4.3 & 39.6 \\
\hline Donation after cardiac death (\%) & 16.0 & 8.4 & $9.9-21.9$ & 0 & 42.4 \\
\hline 25th percentile of wait times (mo) & 15.7 & 8.1 & $10.4-19.2$ & 4.2 & 47.5 \\
\hline Transplant programs in the OPO & 12.8 & 8.9 & 9.0 & $6-15$ & 42 \\
\hline
\end{tabular}

${ }^{a}$ Count (n) of OPOs each year: 2013:40, 2014:51, 2015:51, 2016:51, 2017:48: Average model over the 5 y, that is, not the repeated measures model. 
Nonviable

Kidneys/yr.

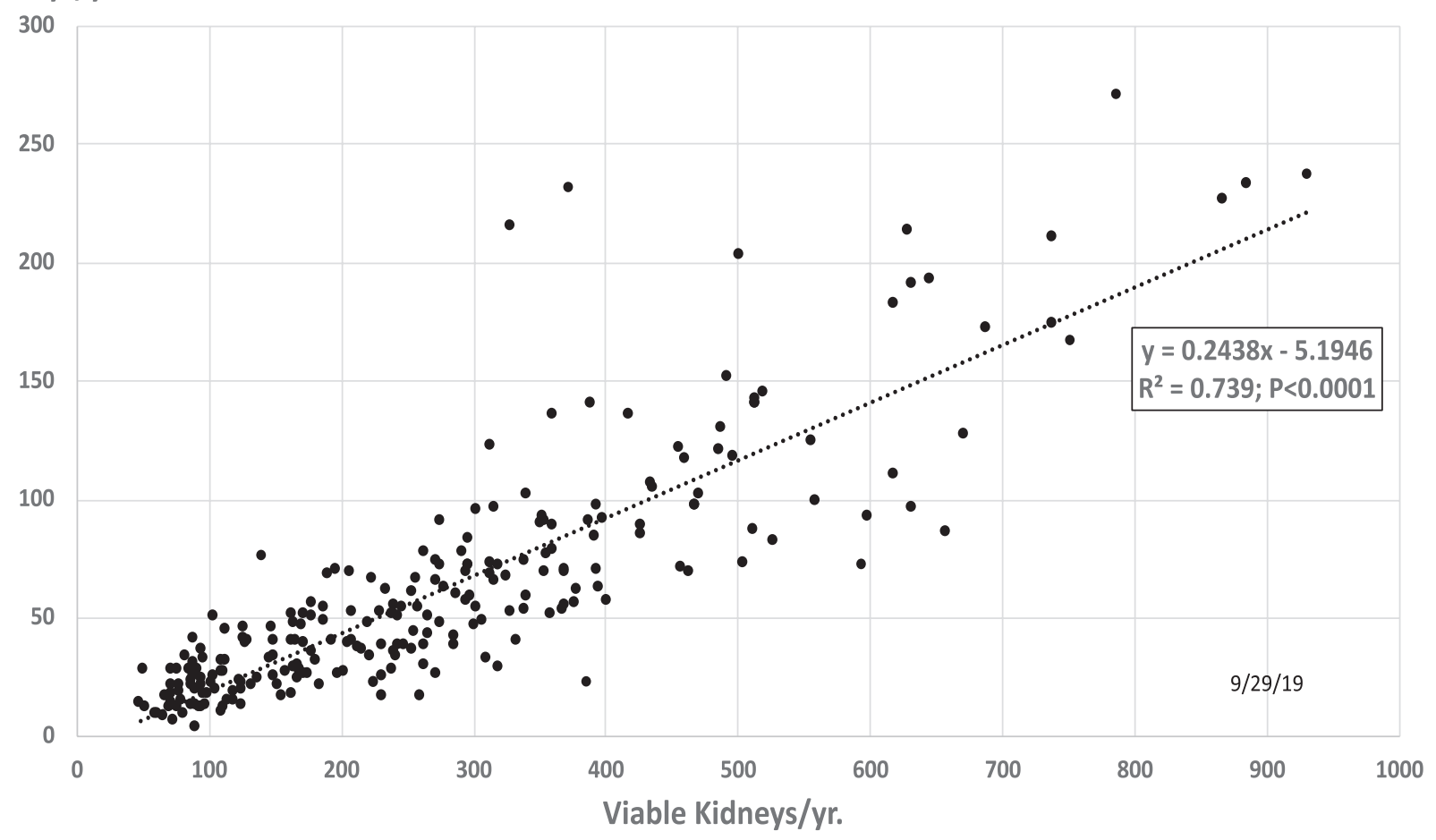

*Dots represent 1 OPO

in 1 year (2013-2017)

FIGURE 1 Correlation between count of viable kidneys and count of nonviable kidneys

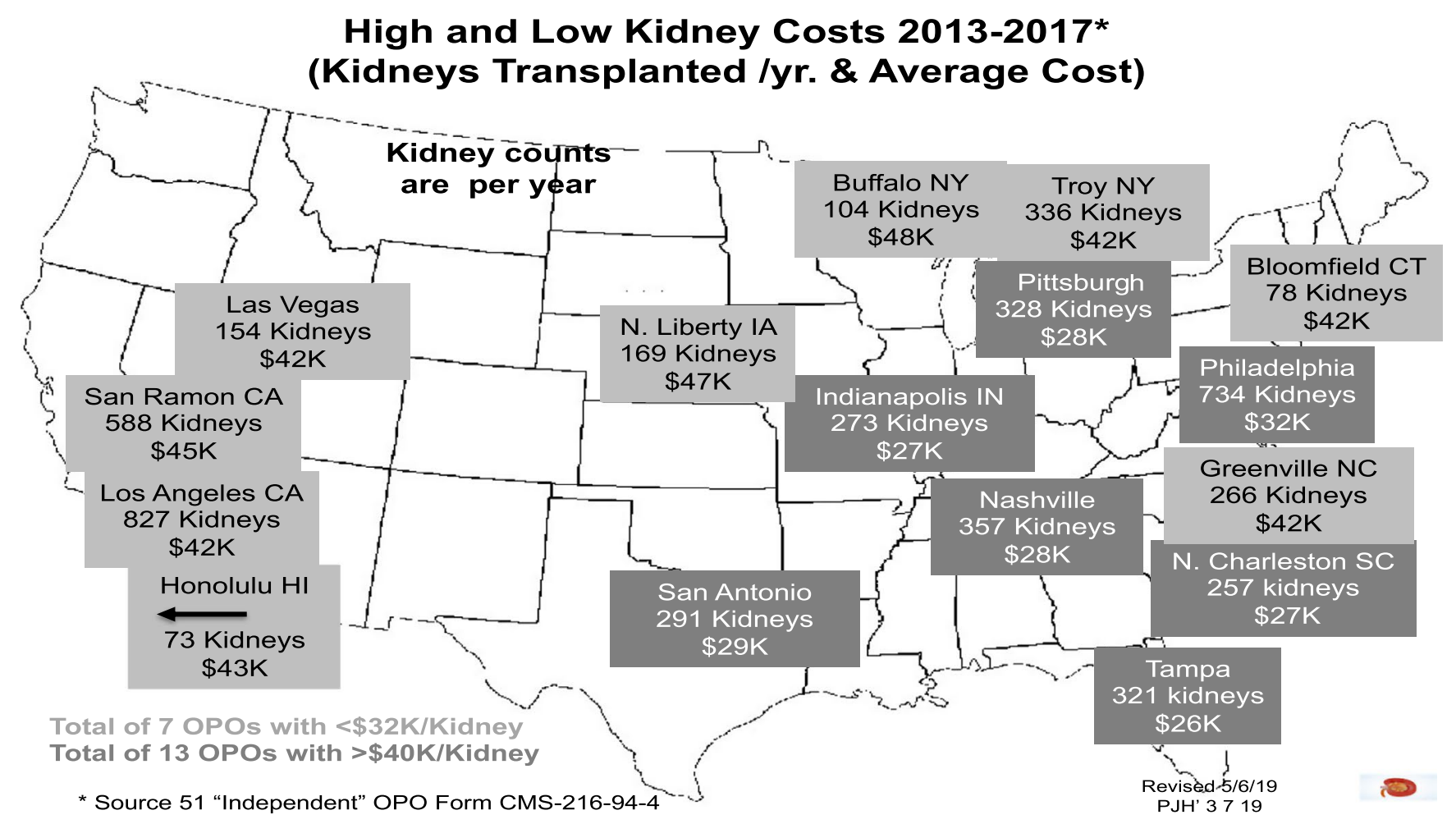

FIGURE 2 Geographic distribution of highest and lowest kidney transplant costs 
OPOs that procure DCD kidneys also have higher cost per viable kidney. As indicated, in Table 2, a 1\% increase in DCD kidneys procured is correlated with higher cost of $\$ 128.53$ per viable kidney $(P<.001)$. At $16 \%$ average DCD kidneys (Table 1$)$ the impact of DCD kidneys would be $16.0 \times \$ 128.53=\$ 2056$. At the average OPO size of 331 total kidneys (viable and nonviable) procured per year, there would be approximately 53 DCD kidneys, which suggests that $\$ 2056 / 53=\$ 39$ increase in the average cost per viable kidney per DCD kidney.

Using the IQR range of SDR of 0.08 (Table 1) and the estimated association between SDR and the cost of a viable kidney of minus $\$ 95.08$ per 0.01 (Table 2) suggests that the difference in cost for a viable kidney comparing for IQR for SDR equals $\$ 760.64$. This means that the estimated average cost of a viable kidney for an OPO at the 25th percentile of SDR (SDR $=0.95$ ) would be $\$ 760.64$ more than a viable kidney from an OPO at the 75th percentile of SDR (SDR $=1.03$ ).

The procurement cost of a transplanted kidney has become more expensive over the 5-year of this study, costing approximately $\$ 1078$ (Table 2), more each year $(P<.0001)$. This would suggest at \$36 102 average cost per viable kidney that the inflation rate in procurement cost per kidney is approximately 3.0\% per year.

Figure 3 displays the quadratic nature of the cost curve in respect to the number of kidneys transplanted per OPO-year. The figure displays the range of viable kidneys from approximately 50-900 kidneys per year. The cost of a kidney declines from the highest estimates for the smallest OPOs to a low point, which ranges between 500 and 600 transplanted kidneys, depending on adjustments. The unadjusted model estimates the highest cost for an OPO with 50 viable kidneys (\$55 995/kidney) to the lowest cost for an OPO with 272 transplants (\$24 289/kidney). In OPOs with >549 transplants, costs began to rise with increasing number of transplants, reaching a cost of \$41 140 for an OPO with 910 transplants. Adjustment for $\mathrm{COL}$, percentage of nonviable kidneys, and percentage of DCD donors changed the shape of the curve slightly, moving the lowest cost per kidney further to the right (OPOs with more kidney transplants per year) and with less cost increase for OPOs with the largest volume of transplants.

There is a computed minimum cost as related to OPO size derived from the estimated quadratic cost parameters (Table 2). Depending on the adjustments to the model, the cost minimum occurs between 500 and 600 viable kidneys per year. The unadjusted model predicts a minimum cost at 549 viable kidneys per year at an average cost of $\$ 33910$.

The model predicts that OPOs that procure more than 549 kidneys experience a cost per kidney higher than the minimum; that is, the cost curve rises after the minimum. And the model predicts that

TAB LE 2 Quadratic estimation of the mean cost per kidney procured 2013-2017

\begin{tabular}{|c|c|c|c|c|c|c|}
\hline \multirow[b]{2}{*}{ OPO measures } & \multicolumn{3}{|l|}{ Full model } & \multicolumn{3}{|c|}{ Parsimonious model $^{\mathrm{a}}$} \\
\hline & $\boldsymbol{\beta}$ & $95 \% \mathrm{Cl}$ & $P$ value & $\boldsymbol{\beta}$ & $95 \% \mathrm{Cl}$ & $P$ value \\
\hline Kidney count/y (per kidney) & -52.63 & -73.64 to -31.62 & $<.0001$ & -39.71 & -57.08 to -22.35 & $<.0001$ \\
\hline $\begin{array}{l}(\text { Kidney count } / y)^{2} \text { (per } \\
\text { kidney) }\end{array}$ & 0.04 & 0.02 to 0.06 & .001 & 0.03 & 0.01 to 0.05 & .005 \\
\hline $\begin{array}{l}\text { Cost of living in the city } \\
\text { where OPO is based (per } \\
1 \text { unit) }\end{array}$ & 100.26 & 24.30 to 176.22 & .01 & 94.89 & 36.75 to 153.03 & .001 \\
\hline Nonviable organs (per 1\%) & 255.07 & 132.02 to 378.11 & $<.0001$ & 274.51 & 176.39 to 372.63 & $<.0001$ \\
\hline $\begin{array}{l}\text { Donation after cardiac death } \\
\text { (per 1\%) }\end{array}$ & 114.07 & 27.37 to 200.77 & .01 & 128.53 & 49.75 to 207.32 & .001 \\
\hline $\begin{array}{l}\text { Standardized donation rate } \\
\text { ratio (per } 0.01 \text { ) }\end{array}$ & -95.23 & -163.63 to -26.83 & .01 & -95.08 & -155.81 to -34.35 & .002 \\
\hline Year (per year) & 1077.86 & 704.58 to 1451.15 & $<.0001$ & 1042.16 & 682.90 to 1401.42 & $<.0001$ \\
\hline $\begin{array}{l}\text { 25th percentile wait time } \\
\text { (per mo) }\end{array}$ & -125.41 & -283.94 to 33.12 & .12 & - & - & - \\
\hline Transplant programs (per 1) & 196.23 & -15.40 to 407.87 & .07 & - & - & - \\
\hline Age > 65 y (per 1\%) & -68.58 & -225.64 to 88.48 & .39 & - & - & - \\
\hline $\begin{array}{l}\text { Land area (per natural log } \\
\text { mile }_{2} \text { ) }\end{array}$ & 392.48 & -671.95 to 1456.90 & .47 & - & - & - \\
\hline White donor (per 1\%) & -45.88 & -99.59 to 7.84 & .09 & & & \\
\hline Male donor (per 1\%) & 34.77 & -44.48 to 114.01 & .39 & & & \\
\hline Total kidneys/donor (per 1) & -1514.11 & -6428.70 to 3400.48 & .55 & & & \\
\hline
\end{tabular}

OPO, organ procurement organization.

${ }^{\mathrm{a}}$ Mean cost per kidney across OPOs $=\$ 36102, \mathrm{n}=234$; Intercept $=-2068400, P<.0001 .9 / 23 / 19$. 


\section{Cost per Viable Kidney}

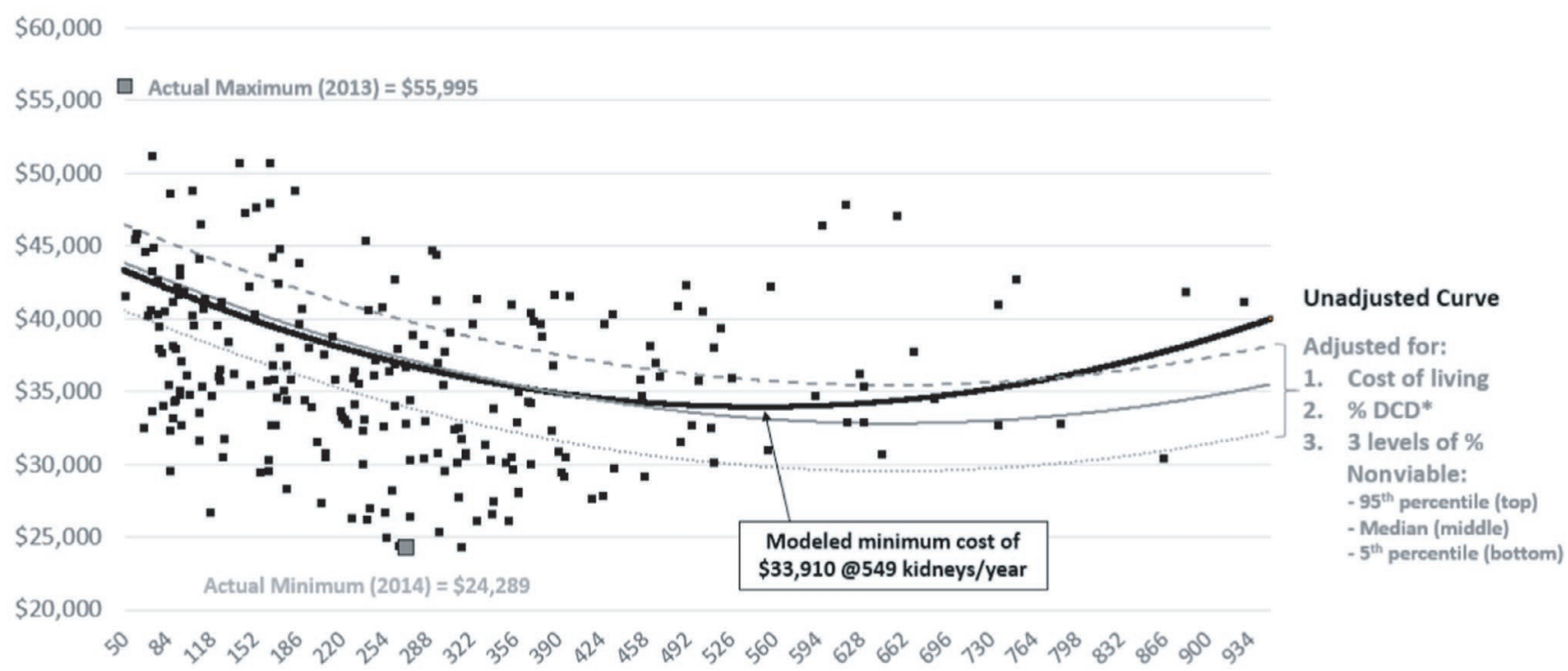

Number of Transplanted Kidneys

\section{(Repeated Measures Model; $\mathbf{n}=238$ )}

FIGURE 3 Cost per viable kidney with selected adjustments

those OPOs recovering fewer than 549 kidneys annually would also experience on average, a cost per kidney higher than the computed minimum.

The small squares in Figure 2 are individual OPO-Yr data points. The estimated effects of the correction for local labor cost (COL) is to shift the minimum cost somewhat to the right (higher procurement levels) and to lower somewhat the increasing cost per average kidney recovered beyond the minimum. When an OPO gains entry into the 70-80th percentile of OPO size, not only do economies of scale disappear, but costs rise.

The total number of kidneys recovered with the intent of transplantation was 81 401, as reported by our dataset. Of these, 66454 underwent transplantation and 18.4\% (14 947) were discarded (nonviable). Because donor age is a strong predictor of kidney discard, ${ }^{14}$ we examined the effect of donor age on viable and nonviable kidneys. There was a correlation between donor age and the percentage of nonviable kidneys $(0.36, P<.01)$ and the donors over the age of $65(0.40, P<.01)$. There was also a correlation with mean number of viable kidneys) and donors over the age of $65(0.18, P<.01)$. We found a correlation with the donors per 1000 deaths less than 75 years for deceased likely to be a donor candidate and the count of viable kidneys $(0.30, P<.04),{ }^{11}$ but not with the SDRR $(0.22, P<.09)$.

\section{4 | DISCUSSION}

For decades, OPO cost reports have been filed with and are available from CMS. Yet, information on OPO costs has generally been scant. There are numerous reports focused on procured organs as well as performance standards, such as ratios of the number of consenting donors to potential donors. ${ }^{15-19}$ Understanding the expenses of organ procurement may shed light on areas in which organ recovery might improve in terms of cost and efficiency. The average cost per kidney and related measures reported in this analysis are based on a robust set of data. Averages were based on 5 years of cost data reported from essentially all US non-hospital based, that is, independent OPOs.

A current issue in kidney transplantation is the discarded, that is, nonviable kidney. Recovery costs for discarded kidneys appear to be built into total kidney procurement costs: total viable and nonviable organ recovery cost divided by the count of viable organs. In effect the costs of nonviable organs (and the cost for the viable organs) are distributed over the OPOs' sum of viable organs. Although it is often implied that Medicare does not pay for nonviable kidneys, the OPO cost forms (Worksheet C) suggest that Medicare does cover OPO costs for such organs.

A second issue with regard to nonviable organs is whether they may be the result of overzealous procurement policies or some other approach to recruiting donors that leads to discard and associated procurement costs. Shown in Figure 1 is a plot and statistical fit of viable vs nonviable organs at the same OPO. There are 241 observations (OPOs by year), which yields a definitive fit to the estimated line. This fitted line has a slope of $0.24(P<.0001)$, which suggests that the count of nonviable organs is a reasonably constant fraction (or percentage) of the viable organs procured. On average for every 10 viable procurements the typical OPO will incur 2.4 nonviable kidneys. The rather high $R^{2}(.74)$ for a cross-section model like this is noteworthy. 
A third issue regarding nonviable kidneys is our suggestion that the small cost of nonviable kidneys often receives undue emphasis compared with the higher value of viable kidneys. It may be that OPOs, in correctly seeking to maximize the number of highly valuable viable kidneys, may also inadvertently increase the number of nonviables. But the great value to society of the former is much larger than the cost of the latter. Earlier research by this team ${ }^{1}$ has shown that the net welfare gain to society from a single viable kidney is about $\$ 1.1$ million and it saves the taxpayer about $\$ 146000$, whereas the cost of producing a nonviable kidney is in the neighborhood of only $\$ 22000$.

Curves depicted in Figure 3 demonstrate that the computed cost per kidney generally follows a quadratic relationship between average cost per kidney and OPO size. The solid black curve is unadjusted by any other covariate. The other curves reflect the basic relationship to size adjusted by different covariates. The curve labeled adjusted for COL generally has little impact in the smaller sizes of the OPO and in the higher ranges of sizes pulls the unadjusted curve downward. This suggests that larger OPOs to the right of the minimum when adjusted for the COL have lower cost when considering the local price levels they face. The three remaining adjusted lines make adjustment for 3 levels of 2 measures of DCD kidneys procured and percentage of nonviable kidneys procured. The three levels of the two selected covariates are 5th percentile, median (50th percentile), and the 95th percentile. The patterns of the $3 \mathrm{ad}-$ justed lines suggest that higher proportions of DCD and nonviable kidneys raise cost.

The estimated coefficient from (Table 2$)$ of $\$ 94.89(P<.002)$ per one unit on the $\mathrm{COL}$ scale indicates that the difference across the IQR of 22 points on the COL index is associated with $22 \times \$ 94.89=\$ 2088$ on the average price per viable kidney. At the overall mean of $\$ 36102$ for price per viable kidney, this IQR spread would represent a difference of $6 \%$ in the average cost per viable kidney. Although this result is quite precise with very narrow uncertainties about estimated parameters, one might observe that $\mathrm{COL}$ differences across OPOs do not explain much of the difference in the cost per kidneys across OPOs. Figure 1, for example, clearly shows a $100 \%$ difference in the OPO reported cost per kidney. Certainly, $\mathrm{COL}$ is clearly a factor in the cost structure of OPO costs but not a very dominant factor.

The cost of organ procurement is relevant to transplant center operations and the relationships that transplant center surgeons and physicians cultivate with the local OPO. Englesbe et al $^{18}$ explain the importance of organ acquisition costs and how clinically oriented physicians and surgeons may benefit from an understanding of transplant center finances. Clinicians should be interested in procuring more organs at lower cost and gaining knowledge about reasonable acquisition costs. An example may be billing acquisition costs in the Kidney Paired Exchange program ${ }^{19}$ in the circumstances of which harbor elements important to both clinical transplantation and its financial foundation. In addition, OPO revenue may be earmarked to address problems shared by donors and recipients, such as quality improvement in organ preservation.

\subsection{Limitations of the study}

We were unable to assess the quality of kidneys procured by the OPOs, although all viable kidneys in the analysis were not only procured but were actually transplanted. Although OPO size, local input prices, nonviable organs (18.4\%), DCD organs (16\%), and year were addressed in determining procurement costs, there are undoubtedly many other contributing factors that simply cannot be fully defined. These include specific geographic characteristics, because any OPO is, by regulation, responsible for a defined geographic area. Thus, the volume and type of potential donor deaths and characteristics of the general population as well as other variables may not be measurable in an analysis such as ours. Labor costs are $50 \%$ of organ procurement expenses, and there is wide variability in labor costs across the United States; ${ }^{9}$ therefore, it is unclear why COL measures do not explain a larger fraction of the variation in procurement costs across OPOs.

We have conducted many quality checks regarding data in the original cost forms, and we have considered the possibility that there may be some double counting of viable kidneys due to transfer of organs among OPOs. Conversations with informed OPO professionals have not affirmed such a possibility. If, however, double counting of viable kidneys is extant, cost estimates per kidney presented in this paper would be biased low. In Appendix S1 we attempt to further explore this matter.

\section{5 | CONCLUSIONS}

Every OPO in the United States is required by CMS to file comprehensive cost reports each year. These reports contain extensive cost detail and provide substantial information on organs and tissue recovered from deceased donors. These data sources can yield useful information when subjected to common analytical methods.

We conclude that costs per deceased donor kidney vary considerably over the 51 independent OPOs included in our analysis, with the highest cost OPOs almost twice as costly as the lowest. Much of this variation in cost is related to the size of operations. In addition, our results confirm that COL does affect cost but only in a modest manner. Other factors shown to affect cost include procurement of nonviable organs, DCD kidneys, and year. We tested a long series of hypotheses in which the null hypothesis of no relationship could not be rejected. In spite of failure to pass the $P$ test these results sill contributed insight into the functions of OPOs.

Many other exact sources of cost variance have yet to be determined. Possible inefficiencies in the current OPO structure might be mitigated by consolidating smaller programs and assessing growth of larger ones ie larger OPOs are frequently operating in the rising cost sector of results. Understanding the reasons for these inefficiencies may help to model more efficient and less costly systems for procurement of donor kidneys and other solid organs. 


\section{DISCLOSURE}

The authors of this manuscript have conflicts of interest to disclose as described by the American Journal of Transplantation. Thomas Peters was retained in a consultant capacity to manage and develop a research program for $\mathrm{DCl}$ Donor Services, a parent organization of three OPOs. The other authors have no conflicts of interest to disclose.

\section{ORCID}

Philip J. Held (iD https://orcid.org/0000-0002-6436-3746

Frank McCormick iD https://orcid.org/0000-0002-6570-8961

\section{DATA AVAILABILITY STATEMENT}

All independent OPOs annually file FORM CMS-216-94, a 25-page cost form, with a federal contractor (Palmetto GPA) located in South Carolina. These data are available under federal law regarding access to public data. The two local cost indices are available via the World Wide Web: https://www.expatistan.com/cost-of-living/index/ north-america\#price-index-explanation and 9. https://www.cms. gov/Medicare/Medicare-Fee-for-Service-Payment/SNFPPS/Wagel ndex.html. 508_compliant_version_of-CMS1605-F_WAGEINDEX transition.csv. Dr Goldberg, University of Pennsylvania has supplied a limited set of data for 1 year.

\section{REFERENCES}

1. Held PJ, McCormick F, Ojo A, Roberts JP. A cost-benefit analysis of government compensation of kidney donors. Am J Transplant. 2016;16:877-885.

2. Matas AJ, Schnitzler M. Payment for living donor (vendor) kidneys: a cost-effectiveness analysis. Am J Transplant. 2003;4:216-221.

3. Nassir BA, Dean CE, Li S, et al. Variation in cost and quality in kidney transplantation. Transplantation. 2015;99(10):2150-2157.

4. Beard TR, Kaserman DL, Osterkamp R. The Global Organ Shortage. Stanford, CA: Stanford University Press; 2013.

5. Public Law 110-144-December 21, 2007. (Charlie Norwood Act, amendments to the National Organ Transplant Act of 1984). http:// optn.transplant.hrsa.gov/policiesAndBylaws/nota.asp. Accessed March 3, 2019.

6. Department of Health and Human Services, Centers for Medicare $\&$ Medicaid Services, Medicare and Medicaid Programs. Conditions for Coverage for Organ Procurement Organizations (OPOs), 42 CFR Parts 413, 441, 486 and 498. Federal Register / Vol. 71, No. 104 / Wednesday, May 31, 2006 / Rules and Regulations.

7. Department of Health and Human Services, Centers for Medicare \& Medicaid Services. Chapter 33 Organ Procurement Organization and Tissue Typing Laboratory Cost Report Form CMS-216-94; Supporting statement for form CMS-216-94 organ procurement organization/ histocompatibility laboratory cost report.

8. https://www.expatistan.com/cost-of-living/index/north-ameri ca\#tprice-index-explanation.

9. https://www.cms.gov/Medicare/Medicare-Fee-for-Service-Payme nt/SNFPPS/Wagelndex.html.508_compliant_version_of-CMS16 05-F_WAGEINDEX_transition.csv.

10. https://www.srtr.org/media/1212/technical_methods_for_osrs_ fall_2016.pdf.

11. Goldberg D, Karp S, Shah MB, Dubay D, Lynch R. Importance of incorporating standardized, verifiable, objective metrics of organ procurement organization performance into discussions about organ allocation. Am J Transplant. 2019. https://doi.org/10.1111/ ajt.15492.

12. Dor A, Held PJ, Pauly MV. The medicare cost of renal dialysis: evidence from a statistical cost function. Med Care. 1992;30:879-891.

13. Greenland S, Senn SJ, Rothman KJ, et al. Statistical tests, P values, confidence intervals, and power: a guide to misinterpretations. Eur J Epidemiol. 2016;31(4):337-350.

14. Marrero WJ, Naik AS, Friedewald JJ, et al. Predictors of deceased donor kidney discard in the United States. Transplantation. 2017;101(7):1690-1697.

15. Guadagnoli E, Christiansen CL, Beasley CL. Potential organ-donor supply and efficiency of organ procurement organizations. Health Care Financ Rev. 2003;24(4):101-110.

16. Goldberg D, Kallan MJ, Fu L, et al. Changing metrics of organ procurement organization performance in order to increase organ donation rates in the United States. Am J Transplant. 2017;17(12):3183-3192.

17. Adler JT, Sethi RK, Yeh H, Markmann JF, Nguyen LL. Market competition influences renal transplantation risk and outcomes. Ann Surg. 2014;260(3):550-557.

18. Englesbe MJ, Ads Y, Cohn JA, et al. The effects of donor and recipient practices on transplant center finances. Am J Transplant. 2008;8:586-592.

19. Updates to Medicare's Organ Acquisition and Donation Payment Policy. April 1, 2016.

\section{SUPPORTING INFORMATION}

Additional supporting information may be found online in the Supporting Information section.

How to cite this article: Held PJ, Bragg-Gresham JL, Peters T, Chertow GM, McCormick F, Roberts JP. The cost of procuring deceased donor kidneys: Evidence from OPO cost reports 2013-2017. Am J Transplant. 2020;20:1087-1094. https://doi.org/10.1111/ait.15669 